\title{
Mobile technology access and use among youth in Nairobi, Kenya: implications for mobile health intervention design
}

\author{
Brenda Kharono $^{1}$, Anne Kaggiah ${ }^{2}$, Cyrus Mugo ${ }^{1,2}$, David Seeh ${ }^{2}$, Brandon L. Guthrie ${ }^{1}$, Megan Moreno ${ }^{3}$, \\ Grace John-Stewart ${ }^{1,4}$, Irene Inwani ${ }^{2}$, Keshet Ronen ${ }^{1 \wedge}$ \\ ${ }^{1}$ Department of Global Health, University of Washington, Seattle, WA, USA; ${ }^{2}$ Department of Paediatrics and Child Health, Kenyatta National \\ Hospital, Nairobi, Kenya; ${ }^{3}$ Department of Pediatrics, University of Wisconsin-Madison, Madison, WI, USA; ${ }^{4}$ Departments of Epidemiology, \\ Medicine, Pediatrics, University of Washington, Seattle, WA, USA \\ Contributions: (I) Conception and design: K Ronen, C Mugo, I Inwani, BL Guthrie, M Moreno, G John-Stewart; (II) Administrative support: C \\ Mugo, A Kaggiah, D Seeh; (III) Provision of study materials or patients: None; (IV) Collection and assembly of data: C Mugo, A Kaggiah, D Seeh, I \\ Inwani; (V) Data analysis and interpretation: B Kharono; (VI) Manuscript writing: All authors; (VII) Final approval of manuscript: All authors. \\ Correspondence to: Keshet Ronen. Acting Assistant Professor, Department of Global Health, University of Washington, Hans Rosling Center, Box \\ 351620, 3980 15th Ave NE, Seattle, WA 98195, USA. Email: keshet@uw.edu.
}

Background: Social media can be used to support the health of underserved youth beyond clinical settings.
Young people are avid users of social media, but estimates of smartphone access among youth in sub-Saharan
Africa are lacking, making it difficult to determine context-appropriateness of online and social media
interventions.

Methods: We conducted a cross-sectional observational survey assessing technology access and use among youth aged 14-24 receiving general outpatient or human immunodeficiency virus (HIV) care in three hospitals in Nairobi, Kenya. Correlates of smartphone access and social media use were evaluated by Poisson regression.

Results: Of 600 youth, 301 were receiving general outpatient care and 299 HIV care. Median age was 18 years. Overall, 416 (69\%) had access to a mobile phone and 288 (48\%) to a smartphone. Of those with smartphones, 260 (90\%) used social media. Smartphone access varied by facility (40\% at the subcounty hospital vs. $55 \%$ at the national referral hospital, $\mathrm{P}=0.004)$ and was associated with older age $[65 \%$ in 20-24-year-old vs. 37\% in 14-19-year-old, adjusted prevalence ratio (aPR) 1.58, 95\% CI: 1.30-1.92], secondary $v$ s. primary education (aPR 2.59, 95\% CI: 1.76-3.81), and HIV vs. general outpatient care (aPR 1.18 , 95\% CI: 1.01-1.38). Social media use was similarly associated with facility, older age, higher education, and male gender.

Conclusions: These data suggest that smartphone-based and social media interventions are accessible in Nairobi, Kenya, in the general population and youth living with HIV, and most appropriate for older youth. Intervention developers and policymakers should consider smartphone and social media interventions as candidates for youth health programs, while noting that heterogeneity of access between and within communities requires tailoring to the specific intervention context to avoid excluding the most vulnerable youth.

Keywords: Youth; smartphone; social media; Kenya; human immunodeficiency virus (HIV)

Received: 12 June 2021; Accepted: 20 October 2021; Published: 20 January 2022.

doi: $10.21037 /$ mhealth-21-23

View this article at: https://dx.doi.org/10.21037/mhealth-21-23

$\wedge$ ORCID: 0000-0001-5625-4884. 


\section{Introduction}

Exponential growth in access to mobile phones over the last decades has spurred development of the field of mobile health (mHealth), defined as the use of mobile devices to support health and healthcare $(1,2)$. While a large body of work exists on using short message service (SMS) text messaging and voice calls for health interventions in high- and lowincome settings (3), expanding access to smartphones and the internet in recent years has provided mHealth platforms with new functionality, such as creation of interactive virtual communities and sharing of multimedia content (4). In high-income countries, young people are known to be early adopters of technology and avid users of social media (defined as interactive electronic communication through which users create online communities and share content, including social networking sites and messaging applications) $(5,6)$. Previous literature-mostly from high-income countries, but also to a lesser extent low- and middle-income countrieshas also shown the feasibility of using smartphone-based and social media interventions with young people to support a variety of health concerns, including sexual and reproductive health, mental health and chronic illness such as HIV and diabetes (7-11).

mHealth interventions have been proposed as an approach to promote health equity, by providing patients with remote support in contexts where barriers exist to inperson care $(12,13)$. This makes them especially appealing in resource-limited settings such as sub-Saharan Africa (SSA). However, such intervention relies on access to the requisite technology platform and communication networks, such as mobile phones (to access SMS and voice calls), computers, or smartphones (to access the internet and social media). Recent data show that access to mobile phones has grown dramatically over the last decade in SSA (1). In Kenya in 2020 , mobile phone penetration among adults was $98 \%$, with $43 \%$ of adults using the internet $(96 \%$ of them on smartphones rather than computers) and $17 \%$ using social media ( $98 \%$ of them on smartphones) (14). Kenya has seen broad uptake of mobile technology, with higher phone penetration than the global average $(67 \%)$ and near-universal use of mobile banking through M-PESA (as of 2016, 96\% of Kenyan households) (15). Internet and social media access remains lower than the global average (59\% and 49\% respectively) (14). Less data exist regarding smartphone and social media access specifically among Kenyan youth under age 25 years. While few studies have used smartphonebased interventions to support the health of youth in SSA, there is growing interest in using this platform in this age group (8,16-19).

While mHealth interventions have the potential to bridge gaps in access to in-person care, use of technology for health interventions also has the potential to perpetuate the "digital divide": unequal access to technology and the internet based on socioeconomic class, race, and gender (20). As formative work to determine whether youth, and in particular youth living with HIV (YLWH) in Nairobi, Kenya, would be served by social media and smartphone interventions, we conducted a study to assess level and correlates of smartphone access and social media use among youth in Nairobi. We present the following article in accordance with the STROBE reporting checklist (available at https:// dx.doi.org/10.21037/mhealth-21-23).

\section{Methods}

\section{Study design}

This cross-sectional study was conducted as formative work for the Vijana-SMART study, which sought to develop a social media intervention for youth in Nairobi.

\section{Study population}

Data collection was conducted between December 2017 and April 2018 at three public hospitals in urban and periurban Nairobi, Kenya, classified by the Ministry of Health as a national referral hospital, a county referral hospital, and a sub-county hospital serving an informal settlement. Given the social determinants of HIV risk, we reasoned that YLWH might have reduced access to technology, which would be important information for HIV-focused mHealth intervention development. In order to evaluate differences in technology access between youth with and without HIV, at each facility, participants were recruited in-person through convenience sampling by study staff in waiting rooms at the general outpatient and HIV care clinics. The general outpatient facilities at the county and sub-county hospitals where we recruited saw patients for simple ailments (e.g., fever, respiratory infections, diarrhea), injuries, and as an entry point for chronic diseases (e.g., diabetes) prior to referral to the specialty clinic. The outpatient facility at the national referral hospital was a youth clinic offering HIV testing, prevention and mental health services. Recruitment was conducted at public healthcare facilities in order to include a diverse 
sample of the general population, including $\mathrm{YLWH}$, in a venue that would inform future facility-based studies. A recruitment target of 100 participants per clinic per facility was set based on feasibility. Participants were eligible for study participation if they were aged 14-24 years, per self-report. This age range was selected as a group with a shared set of challenges to healthcare access. Verbal informed consent to participate was obtained from all participants prior to data collection. Consistent with Kenyan regulations, participation of minors age under 18 years required parental permission and minor assent unless the minor was emancipated by marriage, pregnancy, or parenthood. Additionally, to maximize inclusion and protect confidentiality of those youth whose caregivers were not involved in their medical care, we obtained a waiver of parental permission for minors attending care without an adult caregiver. No financial incentive was provided to participants.

\section{Data collection}

Study staff collected data in-person in a private area at the healthcare facility, using standardized questionnaires administered using a tablet-based electronic system, Open Data Kit (ODK). Participants were given the option of entering responses to sensitive questions on the tablet rather than speaking responses to study staff if they preferred. The questionnaire was collaboratively developed by the study team and pilot tested for comprehension with youth at study facilities prior to data collection. All data were self-reported by participants, and included age, gender, education level, living situation, orphanhood, employment status, and technology access. Household income was assessed but only $30 \%$ of youth provided a response, so these data are not presented. Technology access measures included mobile phone access (owned or shared with others), smartphone access, social media use (Facebook messenger, WhatsApp messenger, Instagram, Snapchat, Telegram, WeChat, Skype, Signal, Viber), Wi-Fi access, airtime spending, and social media use for support with health. The number of participants approached was not recorded, so no measure of survey uptake is reported.

\section{Statistical analysis and variable definitions}

Smartphone access was defined as having access to a phone with a touchscreen (own or shared). Social media use was defined as reporting use of one or more of the following platforms: Facebook, WhatsApp, Instagram, Telegram, Skype, Snapchat, Viber, Signal. Orphanhood was defined as death of a parent before age 18 years. Living situation was defined as a non-exclusive indicator for (I) living with parents/guardians, (II) attending boarding school, and (III) living in multiple places. Financial support was defined as the person(s) who pay for the participants' essential needs, such as food and housing. To account for multiple sources of financial support, it was categorized into the following non-exclusive categories: (I) self, (II) parents/ guardians, (III) other. Correlates of smartphone access were determined using Poisson regression with robust standard errors. This was used in place of logistic regression in order to provide an estimate that more closely approximated risk for common outcomes (21). Univariable analyses were performed for each predictor of interest with the two outcomes of interest (smartphone access and social media use). A multivariable model was performed for each outcome including all variables associated with the outcome in univariable analysis at $\mathrm{P}<0.1$. Data were analyzed using $\mathrm{R}$ version 4.0.2.

\section{Ethics approval}

The study was conducted in accordance with the Declaration of Helsinki (as revised in 2013). The study received approval from the ethical review boards at the University of Washington (study 00002554) and University of Nairobi/Kenyatta National Hospital (study P296/06/2017) and informed consent was taken from all individual participants.

\section{Results}

\section{Participant demographic characteristics}

A total of 600 youth completed the questionnaire, equally distributed between the 3 study facilities and the HIV and outpatient clinics at each. Table 1 summarizes the characteristics of study participants. Median age was 18 years, 359 youth $(59.8 \%)$ were age $14-19$ years and $372(62.0 \%)$ were female. Most $(393,65.5 \%)$ of the participants had completed secondary school. A large majority $(460,76.9 \%)$ of participants lived with their parents or guardians at least some of the time, $109(18.2 \%)$ reported staying in boarding school at least some of the time, and $140(23.4 \%)$ reported living in more than one place. Approximately one-quarter of participants (160, 28.2\%) 
Table 1 Participant characteristics

\begin{tabular}{|c|c|c|c|c|c|c|}
\hline \multirow{2}{*}{ Characteristic } & \multicolumn{2}{|c|}{$\begin{array}{l}\text { Overall } \\
(\mathrm{N}=600)\end{array}$} & \multicolumn{2}{|c|}{$\begin{array}{l}\text { General outpatient care } \\
\qquad(\mathrm{N}=301)\end{array}$} & \multicolumn{2}{|c|}{$\begin{array}{l}\text { HIV care } \\
(\mathrm{N}=299)\end{array}$} \\
\hline & $\mathrm{N}$ & $\%$ & $\mathrm{~N}$ & $\%$ & $\mathrm{~N}$ or median & $\%$ or IQR \\
\hline \multicolumn{7}{|l|}{ Clinical site } \\
\hline National referral hospital & 200 & 33.3 & 100 & 33.2 & 100 & 33.4 \\
\hline Sub-county hospital & 200 & 33.3 & 101 & 33.6 & 99 & 33.1 \\
\hline \multicolumn{7}{|l|}{ Age, years } \\
\hline $14-19$ & 359 & 59.8 & 187 & 62.1 & 172 & 57.5 \\
\hline $20-24$ & 241 & 40.2 & 114 & 37.9 & 127 & 42.5 \\
\hline \multicolumn{7}{|l|}{ Education level } \\
\hline Primary school completed & 112 & 18.7 & 50 & 16.6 & 62 & 20.7 \\
\hline Secondary school completed & 393 & 65.5 & 207 & 68.8 & 186 & 62.2 \\
\hline Above secondary school & 95 & 15.8 & 44 & 14.6 & 51 & 17.1 \\
\hline \multicolumn{7}{|l|}{ Living situation ${ }^{\mathrm{a}, \mathrm{b}}$} \\
\hline With parent(s)/guardian(s)/older relative(s) & 460 & 76.9 & 238 & 79.1 & 222 & 74.7 \\
\hline Boarding school* & 109 & 18.2 & 65 & 21.6 & 44 & 14.8 \\
\hline None (both parents alive up to age 18) & 408 & 71.8 & 246 & 84.2 & 162 & 58.7 \\
\hline \multicolumn{7}{|l|}{ Financial support ${ }^{\mathrm{a}, \mathrm{d}}$} \\
\hline Parent(s)/guardian(s) ${ }^{\star}$ & 353 & 58.9 & 203 & 67.4 & 150 & 50.3 \\
\hline Self (paid work) & 7 & 1.2 & 3 & 1.0 & 4 & 1.3 \\
\hline Other(s) & 240 & 40.1 & 119 & 39.5 & 121 & 40.6 \\
\hline Age at HIV acquisition (years) ${ }^{\mathrm{e}}$ & & & & & 12 & $1-19$ \\
\hline
\end{tabular}

had experienced orphanhood of at least one parent. The majority of participants $(353,58.9 \%)$ reported receiving financial support from their parents/guardians and 240 $(40.1 \%)$ reported financial support from other relatives, friends or partners; only 7 (1.2\%) reported their own paid work supporting their essential needs.

Characteristics were mostly similar between youth enrolled at general outpatient clinics and HIV care clinics. 
Table 2 Technology access and use

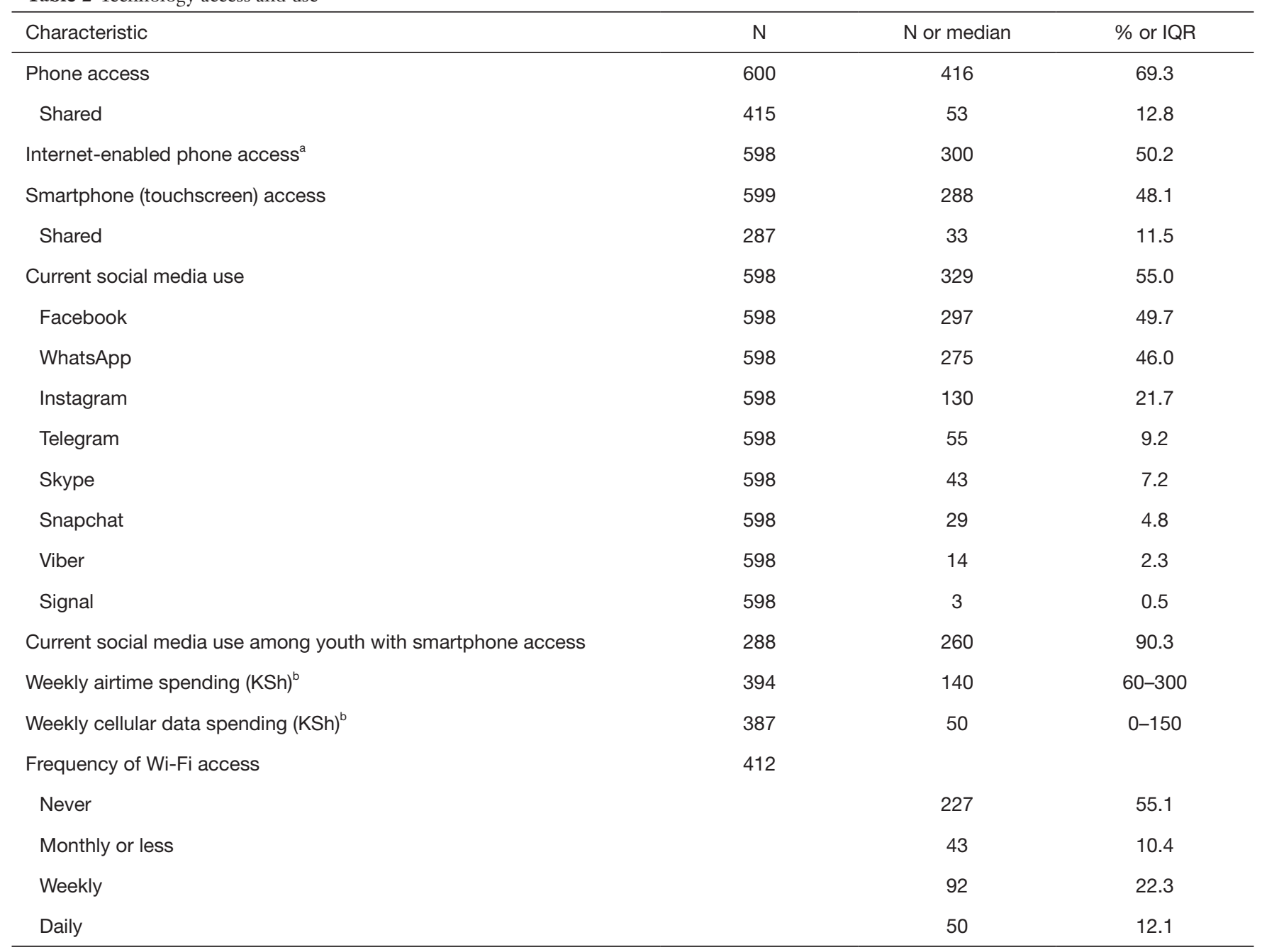

a, includes non-smartphone "feature" phones with internet access; ${ }^{b}, 100 \mathrm{KSh}=1$ USD. IQR, interquartile range.

However, the prevalence of orphanhood was significantly higher among YLWH at HIV care clinics: almost half (114, 41.3\%) of YLWH had experienced orphanhood, compared with $15.8 \%$ [46]. Fewer YLWH were financially supported by their parents or guardians than youth attending general outpatient care $[150(50.3 \%)$ vs. 203 (67.4\%)] and fewer attended boarding school [44 (14.8\%) vs. 65 (21.6\%)]. Among YLWH, median age at HIV acquisition was 12 [interquartile range (IQR), 1-19 years].

\section{Technology access and use}

Youth's access to and use of mobile technology is summarized in Table 2. The majority of youth (416, 69.3\%) had access to a mobile phone. Around half had access to a smartphone $(288,48.1 \%)$ or an internet-enabled "feature" phone $(300,50.2 \%)$. One-tenth $(33,11.5 \%)$ of youth with smartphone access shared it with another person. A little over half of all youth $(329,55.0 \%)$ and most youth with smartphone access $(260,90.3 \%)$ used social media, most commonly Facebook or WhatsApp. Weekly spending on airtime and cellular data was a median of $140 \mathrm{KSh}(1.40$ USD) and $50 \mathrm{KSh}(0.50 \mathrm{USD})$ respectively. A little under half $(185,44.9 \%)$ had access to $\mathrm{Wi}-\mathrm{Fi}$, with 122 (29.6\%) having access weekly or more.

\section{Correlates of smartphone access}

Table 3 summarizes the association between participant characteristics and access to a smartphone. Univariable 
Table 3 Correlates of smartphone access

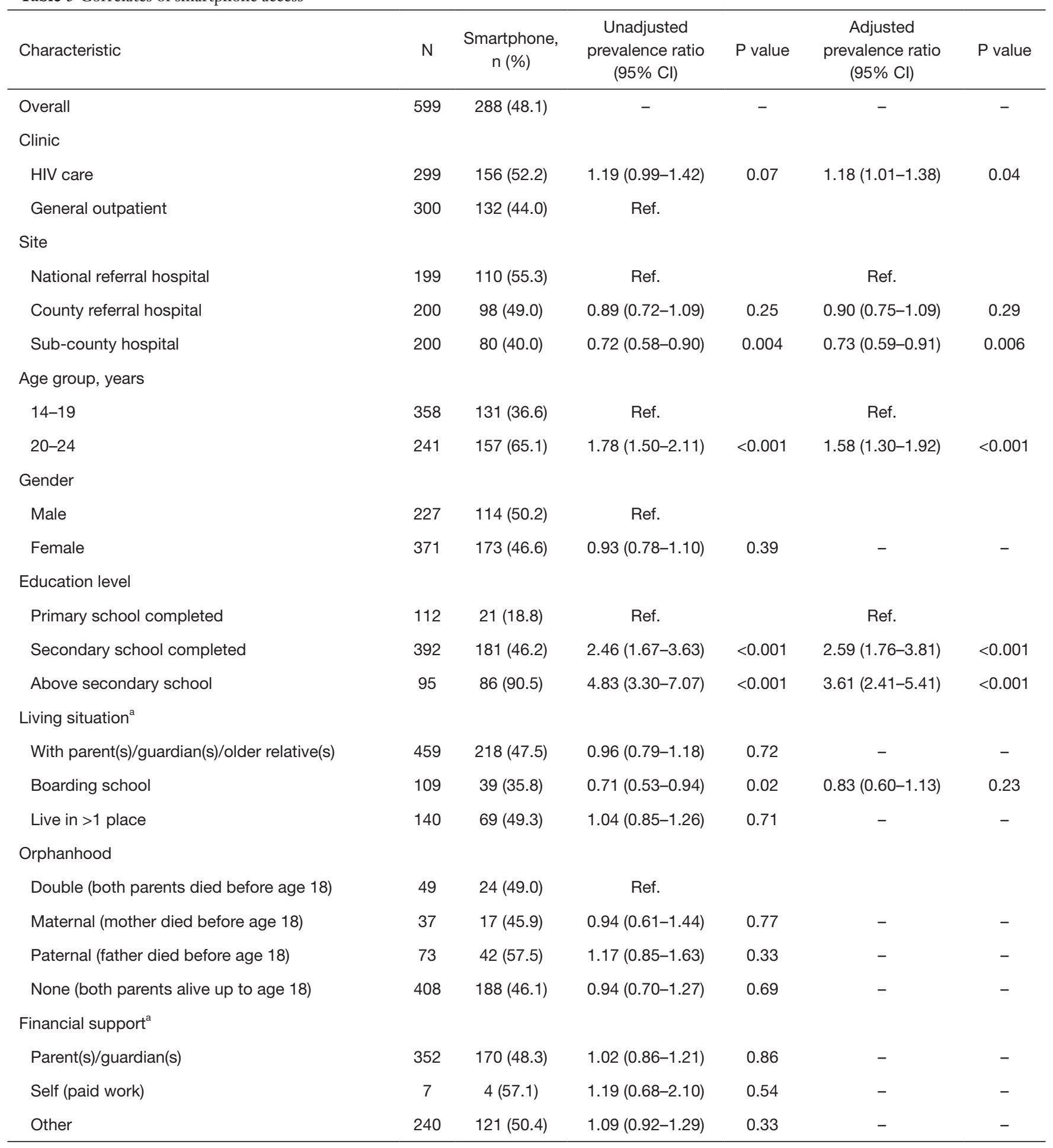

a , living situation and financial support options are not mutually exclusive; participants could choose more than 1 option. Cl, confidence interval. 
analyses indicated that smartphone access varied significantly by clinic site: youth at the referral hospital had the highest access $(55.3 \%)$ while youth at the sub-county hospital had the lowest $[40.0 \%$, prevalence ratio (PR) $0.72,95 \%$ confidence interval (CI): 0.58-0.90]. Smartphone access was higher among 20-24-year-old than 14-19-year-old (65.1\% vs. $36.6 \%$, PR 1.78, 95\% CI: $1.50-2.11$ ). Smartphone access was also associated with more advanced education: $18.8 \%$ of youth who had completed primary school had smartphone access compared with $46.2 \%$ of those who had completed secondary school and $90.5 \%$ of those who had beyond secondary school education [PR 2.46 (95\% CI: 1.67-3.63) and 4.83 (95\% CI: 3.30-7.07)]. Youth who attended boarding school were less likely to have access to a smartphone [PR 0.71 (95\% CI: 0.53-0.94)]. There was a trend for higher smartphone access among youth attending HIV care $v s$. general outpatient care. We found no significant association between smartphone access and gender, orphanhood, or source of financial support.

In multivariable analysis, participant age, education level, and facility remained associated with smartphone access. Participants age 20-24 were 1.58 times as likely to have smartphone access (95\% CI: $1.30-1.92)$ as younger participants; participants who had completed secondary school were 2.59 times as likely to have access as participants who had completed primary school (95\% CI: 1.76-3.81), with a more pronounced effect among those who had above secondary $v s$. primary education (PR 3.61, 95\% CI: 2.41-5.41); and participants at the sub-county hospital were 0.73 times as likely to have access as participants from the national referral hospital (95\% CI: 0.59-0.91). Additionally, in multivariable analysis YLWH were found to have significantly higher smartphone access (PR 1.18, 95\% CI: 1.01-1.38). Given the differences we observed by facility, we conducted analyses stratified by site. All effect estimates were consistent in stratified analyses, though the association with HIV status became statistically non-significant when data were stratified (data not shown).

\section{Correlates of social media use}

Table 4 summarizes correlates of social media use. Correlates of social media use were similar to those of smartphone access. We found social media use differed significantly by facility, ranging from $67.8 \%$ in the referral hospital to $44.0 \%$ in the sub-county hospital, and was significantly higher among YLWH than those attending general outpatient care (aPR 1.29, 95\% CI: 1.12-1.48).
Social media use was higher among youth age 20-24 than those age 14-19 (aPR 1.35, 95\% CI: 1.15-1.59) and those with secondary and post-secondary education compared with those who had completed primary education (secondary education aPR 2.56, 95\% CI: 1.79-3.66; post-secondary education aPR 3.49, 95\% CI: 2.41-5.06). Additionally, female youth were less likely to use social media than male youth (aPR 0.85, 95\% CI: 0.74-0.97).

\section{Discussion}

\section{Summary of findings}

This study reports levels and determinants of access to smartphones in youth age 14-24 living with and without HIV in Nairobi, Kenya. We found that, while almost $70 \%$ of youth across 3 facilities had access to a phone, a little under half had access to a smartphone. Among those who had smartphone access, the vast majority (90\%) used social media, predominantly Facebook and WhatsApp. Cellular data usage was low and a little less than half of youth had access to Wi-Fi. We found significant variation in smartphone access between different facilities in Nairobi, and that smartphone access and social media use were associated with older age (age 20-24 vs. 14-19 years) and higher education, even when adjusting for other demographic characteristics. We also found that YLWH had higher smartphone access and social media use than youth attending general outpatient care and that men had higher social media use than women.

\section{Comparison with previous work}

Our findings add to the limited data on technology access by youth in SSA and highlight the need for studies that evaluate reach of technology-based interventions in specific communities. Most available data in SSA are from national online surveys of adults only, administered by industry groups (14). The January 2018 Hootsuite report found that $43 \%$ of Kenyan adults at the time were using smartphones and $14 \%$ of the total Kenyan population was using social media (22). Our analysis, in contrast, focused on adolescents and young adults, who are understudied and have unique health needs and barriers to healthcare (23). Moreover, our study population included participants seeking medical care in informal settlements in Nairobi, expanding the relevance to lower income communities.

Our findings are consistent with studies conducted 
Table 4 Correlates of social media use

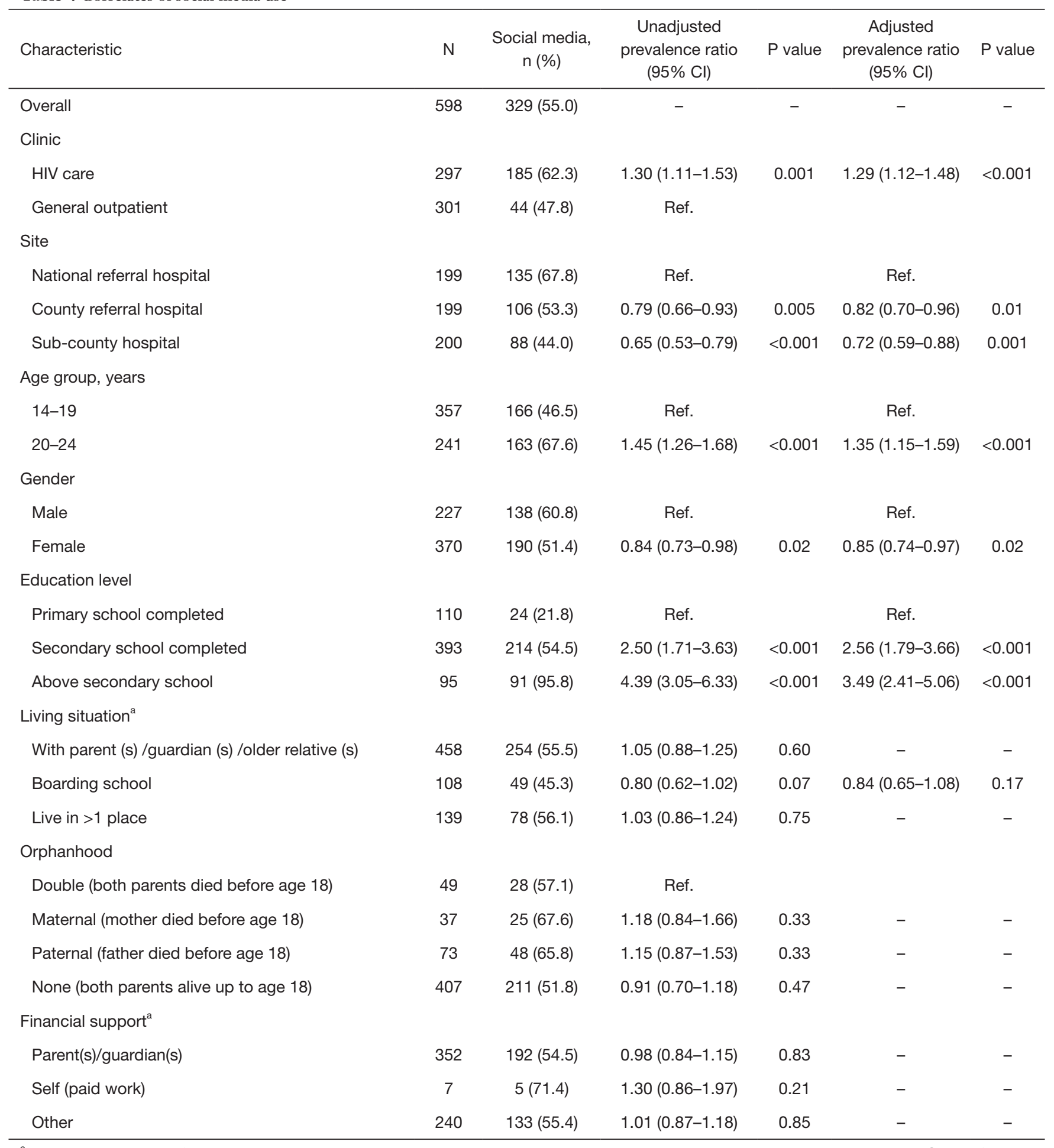

a , living situation and financial support options are not mutually exclusive; participants could choose more than 1 option. Cl, confidence interval. 
between 2014 and 2020 in other parts of SSA. A 2018 study among youth age 13-24 years in Zimbabwe reported 67\% phone access, $57 \%$ smartphone access, and higher phone and internet access among older, more highly educated, and male youth (24). A 2014-2016 study among youth aged 18-24 years living with HIV in Uganda reported 94\% owned a phone and $37 \%$ owned a smartphone, with higher internet use among older youth and men (25). A 2020 study among mothers aged 16-19 years in Nigeria reported $90 \%$ owned a phone and $21 \%$ owned a smartphone; correlates of ownership were not reported (26). A 2017 report on smartphone ownership and internet access among adults in SSA reported that both were associated with higher education and male gender (27). To our knowledge, no previous studies have compared technology access among youth with $v s$. without HIV.

\section{Interpretation and implications for intervention design}

Given that access to technology is a prerequisite for individuals to benefit from mHealth interventions, our findings provide critical information to inform development of responsive and accessible mHealth interventions for youth in Nairobi. Additionally, our analysis of the correlates of smartphone access and social media use allows understanding of which subgroups of youth are reached and excluded by social media and smartphone-based interventions, and evaluation of the equity implications of pursuing these interventions. Our findings indicate that smartphone-based interventions, including interventions accessed on websites, web applications, mobile applications and social media, would be accessible to approximately half of youth aged 14-24 years in this context, approximately two-thirds of those aged 20-24 years, and $90 \%$ of those with above secondary education.

The association with age is likely explained by the fact that phone access is typically sought as individuals gain independence, mobility, and financial resources. Additionally, in univariable analysis we found an association between boarding school attendance and lower smartphone access. Though the proportion of youth in our study attending boarding school was relatively low, boarding school attendance is common in Kenya and elsewhere in SSA and has been documented as a barrier to accessing healthcare (28). Students are typically not allowed access to their phones while in staying at boarding school, which may contribute to lower access in adolescents still in school. mHealth interventions focused on school-age adolescents should consider unique needs and access barriers in boarding schools.

The association with higher education (adjusting for age) highlights the importance of socioeconomic status. Education level is an important component of socioeconomic status, so this finding suggests that within our study population, smartphones remain more difficult to access for youth with lower socioeconomic status. This has important implications for the equity and impact of health interventions in this context. Socioeconomic status is a universal predictor of health and access to healthcare (29), so an intervention channel that is preferentially accessible to youth with higher socioeconomic status may systematically exclude the youth with the greatest need, limiting the intervention's public health impact.

We detected significant variation in smartphone access by healthcare facility within Nairobi, with lowest access at the lowest level (sub-county) facility and highest at the highest level (national referral) facility. Facility-level differences may be driven by differences in socioeconomic status of patients receiving care at different facility levels, or by other contextual differences in different parts of Nairobi. Although individual-level associations we detected were consistent across facilities, this observation highlights the need for targeted formative work in the local context of an intervention to determine its reach and generalizability. In resource-limited settings, mHealth interventions are often delivered by patients self-registering or being registered by a healthcare worker while in healthcare facility waiting areas (30). In such a delivery model, broad access to the intervention platform is critical.

Finally, we found that youth seeking HIV care had higher smartphone access and social media use than youth attending general care. Given the higher prevalence of orphanhood among YLWH, higher phone access might be explained by YLWH having greater independence and responsibility than other youth. This finding is reassuring for developers of mHealth interventions for $\mathrm{YLWH}$, in that it indicates there are no systematic access barriers unique to YLWH.

Technology uptake has increased exponentially over the last decade, including in Kenya $(31,32)$ and access is expected to continue growing over the coming years. Development of forward-thinking, evidence-based interventions using platforms that are expected to be widely accessible within a few years is valuable despite current access limitations. However, it is critical for intervention developers to use smartphone-based interventions 
judiciously to avoid perpetuating health inequities. This means ensuring the sub-populations that stand to benefit from interventions have access to them and considering alternative approaches and differentiated care models based on variation in individual access. This may include developing interventions that can be interchangeably delivered through social media, basic mobile phone technology such as SMS, or in-person modalities.

\section{Limitations}

While our study provides valuable data on access to mobile technology in youth in Nairobi, it represents a snapshot in late 2017-early 2018. Evaluations over the coming years will likely demonstrate higher access, particularly in light of the introduction of social distancing and remote learning for Kenyan students during the COVID-19 pandemic (33). While students were not provided devices, online educational material and reduced cost of mobile data were provided as part of Kenya's COVID-19 response (34). Additionally, we conducted this study only in Nairobi, which, as the capital city, likely has higher technology access and may not be representative of other parts of Kenya. We also used clinicbased recruitment so our data cannot be generalized to youth who do not attend medical care. While we described cellular data use and Wi-Fi access, we did not assess frequency or amount of time using the internet or social media. Access to cellular data or Wi-Fi may be a limiting factor in using online interventions, and our questionnaire was not designed to determine participants' ability to access online interventions beyond their baseline data use. More detailed data are needed to understand how frequently and readily youth connect to the internet, and whether provision of data bundles is needed to deliver a social media intervention in this context. Finally, this study focused only on measuring access; future studies should explore the nature of barriers experienced by youth wishing to access social media, and their experiences of using social media interventions. Data on the feasibility and attractiveness of the Vijana-SMART social media intervention among YLWH in Nairobi are presented elsewhere (35).

\section{Conclusions}

In conclusion, this study is one of a small number exploring access to smartphones and social media among youth in SSA and thus has important implications for development of youth-specific mHealth interventions. Our data suggest that such interventions are more appropriate for older youth, and that heterogeneity of access across communities likely requires tailoring to the specific intervention context, including development of digital and non-digital delivery modalities to maximize reach. Importantly, even among older youth, access was not universal and was associated with higher education level, highlighting that smartphonebased interventions in this group may exclude youth of lower socioeconomic status. Given the rapid growth of mobile technology and the timeline from an intervention's inception to its deployment, forward-thinking investigation of smartphone-based interventions is warranted despite current access limitations. However, it will be critical to examine whether the access gaps identified in this study narrow or persist in years to come and assess equity of social media interventions.

\section{Acknowledgments}

We wish to thank the Vijana-SMART participants and clinic staff at the 3 study sites.

Funding: This work was supported by the University of Washington's Center for AIDS Research New Investigator Award (National Institutes of Health grant number P30AI027757). Authors were also supported by National Institutes of Health grants D43TW009580 to CM, K24HD054314 to GJS, K18MH122978 to KR, and the University of Washington's Global Center for Integrated Health of Women, Adolescents, and Children (Global WACh).

\section{Footnote}

Reporting Checklist: The authors have completed the STROBE reporting checklist. Available at https://dx.doi. org/10.21037/mhealth-21-23

Data Sharing Statement: Available at https://dx.doi. org/10.21037/mhealth-21-23

Peer Review File: Available at https://dx.doi.org/10.21037/ mhealth-21-23

Conflicts of Interest: All authors have completed the ICMJE uniform disclosure form (available at https://dx.doi. org/10.21037/mhealth-21-23). CM was supported by National Institutes of Health grant D43TW009580. BLG declares funding from National Institutes of 
Health (grant P30AI027757). GJS declared funding from National Institutes of Health (grants K24HD054314 and P30AI027757). KR declares funding from National Institutes of Health (grants K18MH122978 and P30AI027757). The other authors have no conflicts of interest to declare.

Ethical Statement: The authors are accountable for all aspects of the work in ensuring that questions related to the accuracy or integrity of any part of the work are appropriately investigated and resolved. The study was conducted in accordance with the Declaration of Helsinki (as revised in 2013). The study was approved by the University of Washington (study 00002554) and University of Nairobi/Kenyatta National Hospital (study P296/06/2017) institutional review boards and informed consent was taken from all individual participants.

Open Access Statement: This is an Open Access article distributed in accordance with the Creative Commons Attribution-NonCommercial-NoDerivs 4.0 International License (CC BY-NC-ND 4.0), which permits the noncommercial replication and distribution of the article with the strict proviso that no changes or edits are made and the original work is properly cited (including links to both the formal publication through the relevant DOI and the license). See: https://creativecommons.org/licenses/by-nc-nd/4.0/.

\section{References}

1. World Bank. Digital Dividends: World Development Report 2016. 2016.

2. World Health Organization. Global Strategy on Digital Health 2020-2024 [Internet]. 2019. Available online: https://extranet.who.int/dataform/upload/surveys/183439/ files/Draft Global Strategy on Digital Health.pdf

3. Marcolino MS, Oliveira JAQ, D'Agostino M, et al. The Impact of mHealth Interventions: Systematic Review of Systematic Reviews. JMIR Mhealth Uhealth 2018;6:e23.

4. Moreno MA, D'Angelo J. Social Media Intervention Design: Applying an Affordances Framework. J Med Internet Res 2019;21:e11014.

5. Pew Research Center. Teens, social media and technology 2018. 2018.

6. Moreno MA, D'Angelo J, Whitehill J. Social Media and Alcohol: Summary of Research, Intervention Ideas and Future Study Directions. Media Commun 2016;4:50-9.

7. Grist R, Cliffe B, Denne M, et al. An online survey of young adolescent girls' use of the internet and smartphone apps for mental health support. BJPsych Open 2018;4:302-6.

8. Ronen K, Grant E, Copley C, et al. Peer Group Focused eHealth Strategies to Promote HIV Prevention, Testing, and Care Engagement. Curr HIV/AIDS Rep 2020;17:557-76.

9. Yonker LM, Zan S, Scirica CV, et al. "Friending" teens: systematic review of social media in adolescent and young adult health care. J Med Internet Res 2015;17:e4.

10. Martin P, Cousin L, Gottot S, et al. Participatory Interventions for Sexual Health Promotion for Adolescents and Young Adults on the Internet: Systematic Review. J Med Internet Res 2020;22:e15378.

11. Fedele DA, Cushing CC, Fritz A, et al. Mobile Health Interventions for Improving Health Outcomes in Youth: A Meta-analysis. JAMA Pediatr 2017;171:461-9.

12. Ben-Zeev D, Atkins DC. Bringing digital mental health to where it is needed most. Nat Hum Behav 2017;1:849-51.

13. Holeman I, Kane D. Human-centered design for global health equity. Inf Technol Dev 2019;26:477-505.

14. Hootsuite. Digital in 2020 - Kenya [Internet]. 2020. Available online: https://datareportal.com/reports/digital2020-kenya

15. Suri $T$, Jack $W$. The long-run poverty and gender impacts of mobile money. Science 2016;354:1288-92.

16. Pfeiffer C, Kleeb M, Mbelwa A, et al. The use of social media among adolescents in Dar es Salaam and Mtwara, Tanzania. Reprod Health Matters 2014;22:178-86.

17. Winskell K, Sabben G, Akelo V, et al. A Smartphone Game-Based Intervention (Tumaini) to Prevent HIV Among Young Africans: Pilot Randomized Controlled Trial. JMIR Mhealth Uhealth 2018;6:e10482.

18. Visser M, Kotze M, van Rensburg MJ. An mHealth HIV prevention programme for youth: lessons learned from the iloveLife.mobi programme in South Africa. AIDS Care 2020;32:148-54.

19. Henny KD, Wilkes AL, McDonald CM, et al. A Rapid Review of eHealth Interventions Addressing the Continuum of HIV Care (2007-2017). AIDS Behav 2018;22:43-63.

20. Wood BR, Young JD, Abdel-Massih RC, et al. Advancing Digital Health Equity: A Policy Paper of the Infectious Diseases Society of America and the HIV Medicine Association. Clin Infect Dis 2021;72:913-9.

21. Zou G. A modified poisson regression approach to prospective studies with binary data. Am J Epidemiol 2004;159:702-6.

22. Hootsuite. Digital in 2018 - Kenya. Available online: 
https://datareportal.com/reports/digital-2018-kenya

23. Patton GC, Sawyer SM, Santelli JS, et al. Our future: a Lancet commission on adolescent health and wellbeing. Lancet 2016;387:2423-78.

24. Doyle AM, Bandason T, Dauya E, et al. Mobile Phone Access and Implications for Digital Health Interventions Among Adolescents and Young Adults in Zimbabwe: Cross-Sectional Survey. JMIR Mhealth Uhealth 2021;9:e21244.

25. Wanyama JN, Nabaggala SM, Kiragga A, et al. High mobile phone ownership but low internet access and use among young adults attending an urban HIV clinic in Uganda. Vulnerable Child Youth Stud 2018;13:207-20.

26. Kola L, Abiona D, Adefolarin AO, et al. Mobile Phone Use and Acceptability for the Delivery of Mental Health Information Among Perinatal Adolescents in Nigeria: Survey Study. JMIR Ment Health 2021;8:e20314.

27. Silver L, Johnson C. Internet Connectivity Seen as Having Positive Impact on Life in Sub-Saharan Africa but Digital Divides Persist [Internet]. 2018. Available online: https:// www.pewresearch.org/global/2018/10/09/internetconnectivity-seen-as-having-positive-impact-on-life-insub-saharan-africa/

28. Mutwa PR, Van Nuil JI, Asiimwe-Kateera B, et al. Living situation affects adherence to combination antiretroviral therapy in HIV-infected adolescents in Rwanda: a qualitative study. PLoS One 2013;8:e60073.

29. Kachmar AG, Connolly CA, Wolf S, Curley MAQ. Socioeconomic Status in Pediatric Health Research: A Scoping Review. J Pediatr [Internet]. 2019;213:16370. Available online: https://doi.org/10.1016/

doi: $10.21037 /$ mhealth-21-23

Cite this article as: Kharono B, Kaggiah A, Mugo C, Seeh D, Guthrie BL, Moreno M, John-Stewart G, Inwani I, Ronen K. Mobile technology access and use among youth in Nairobi, Kenya: implications for mobile health intervention design. mHealth 2022;8:7. j.jpeds.2019.06.005

30. Barron P, Peter J, LeFevre AE, et al. Mobile health messaging service and helpdesk for South African mothers (MomConnect): history, successes and challenges. BMJ Glob Health 2018;3:e000559.

31. International Telecommunication Union. World Telecommunication/ICT Indicators Database [Internet]. 2021. Available online: http://handle.itu.int/11.1002/ pub/81733fd9-en

32. Communications Authority of Kenya. Third quarter sector statistics report for the financial year 2019/2020 (January - March 2020) [Internet]. Nairobi; 2020 [cited 2020 Oct 4]. Available online: https://ca.go. ke/wp-content/uploads/2020/07/Sector-StatisticsReport-Q3-2019-2020-.pdf

33. Kenyan Ministry of Education. Are Children learning during COVID-19? Findings of the Rapid Survey on Education Innovations in Kenya [Internet]. 2020. Available online: https://kicd.ac.ke/broadcast-schedule/rapid-surveyon-education-innovations-in-kenya-2020/

34. Kenyan Ministry of Education. Kenya Basic Education Covid-19 Emergency Response Plan State Department of Early Learning and Basic Education [Internet]. 2020. Available online: https://www.education.go.ke/downloads/ file/798-emergency-response-plan

35. Karusala N, Seeh DO, Mugo C, Guthrie B, Moreno MA, John-Stewart G, et al. "That courage to encourage": Participation and Aspirations in Chat-based Peer Support for Youth Living with HIV. In: Proceedings of the 2021 CHI Conference on Human Factors in Computing Systems, New York, NY, USA: ACM, 2021:1-17. 